\title{
Efecto de los polisacáridos sulfurados marinos en los parámetros productivos y respuesta celular en pollo de engorda
}

\section{Effect of marine sulfurized polysaccharides on productive parameters, and cellular response in broilers}

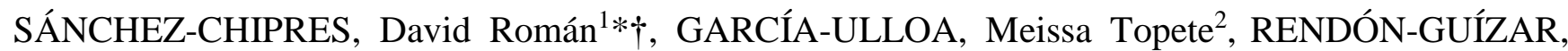 \\ Jesús Ignacio ${ }^{1}$ y RAMÍREZ-ACOSTA, Mariana ${ }^{1}$ \\ ${ }^{1}$ Universidad de Guadalajara, Departamento de Producción Animal, Centro Universitario de Ciencias Biológicas y \\ Agropecuarias \\ ${ }^{2}$ Olmix SA de CV
}

ID 1 ${ }^{\mathrm{er}}$ Autor: David Román, Sánchez-Chipres / ORC ID: 0000-002-5273-0393, CVU CONACYT ID: 69431

ID $1^{\text {er }}$ Coautor: Meissa Topete, García-Ulloa / ORC ID: 0000-002-2277-4731

ID $2^{\text {do }}$ Coautor: Jesús Ignacio, Rendón-Guízar / ORC ID: 0000-0002-2092-6391, CVU CONACYT ID: 696040

ID $3^{\text {er }}$ Coautor: Mariana, Ramírez-Acosta / ORC ID: 0000-0002-3560-9057, CVU CONACYT ID: 696054

DOI: $10.35429 / J C P E .2019 .21 .6 .35 .39$

Recibido 09 Agosto, 2019; Aceptado 30 Noviembre, 2019

\begin{abstract}
Resumen
Objetivos: El objetivo del trabajo fue identificar una alternativa natural que permita obtener parámetros productivos más eficientes y que a su vez el sistema inmunológico de las aves sea más resistente a los patógenos. Se ha demostrado que la administración de polisacáridos de alga marina promueve una mayor resistencia a los individuos sometidos a condiciones de estrés, sugiriendo un efecto inmunoestimulante. Metodología: Se utilizaron 582 pollos machos de línea genética Cobb, con un día de nacidos los cuales fueron distribuidos en 2 tratamientos con 3 repeticiones de 97 aves: tratamiento control y tratamiento con polisacáridos. Los polisacáridos utilizados fueron de la marca Olmix $\odot$ y se utilizaron a una dosis de $45 \mathrm{~g}$ por cada $500 \mathrm{~L}$ de agua los días 9, 11, 12, 19, 21, 22, 34, 36, y 37 de vida. Se determinaron parámetros productivos y respuesta celular, la cual fue realizada mediante frotis teñido con la técnica hayem-turk para posteriormente realizar el conteo en la cámara de neubauer. Los muestreos para el conteo de leucocitos y linfocitos fueron realizados los días 9, 12, 19, 22, 34 y 37. Contribución: La utilización de polisacáridos puede favorecer la ganancia de peso del pollo de engorda.
\end{abstract}

Polisacáridos, Leucocitos, Linfocitos

\begin{abstract}
Objectives: The objective of the work was to identify a natural alternative that allows to obtain more efficient productive parameters and that in turn the immune system of the birds is more resistant to pathogens. The administration of seaweed polysaccharides has been shown to promote greater resistance to individuals under stress conditions, suggesting an immunostimulatory effect. Methodology: 582 male chickens of the Cobb genetic line were used, with one day of births which were distributed in 2 treatments with 3 repetitions of 97 birds: control treatment and treatment with polysaccharides. The polysaccharides used were of the Olmix (C) brand and were used at a dose of $45 \mathrm{~g}$ per $500 \mathrm{~L}$ of water on days $9,11,12,19,21,22$, 34, 36, and 37 of life. Productive parameters and cellular response were determined, which was performed by smears stained with the hayem-turk technique to later perform the count in the neubauer chamber. Samples for leukocyte and lymphocyte count were performed on days $9,12,19,22,34$ and 37 . Contribution: The use of polysaccharides can favor the weight gain of the broiler.
\end{abstract}

Polysaccharides, Leukocytes, Lymphocytes

Citación: SÁNCHEZ-CHIPRES, David Román, GARCÍA-ULLOA, Meissa Topete, RENDÓN-GUÍZAR, Jesús Ignacio y RAMÍREZ-ACOSTA, Mariana. Efecto de los polisacáridos sulfurados marinos en los parámetros productivos y respuesta celular en pollo de engorda. Revista de Energía Química y Física. 2019. 6-21: 35-39

\footnotetext{
* Correspondencia al Autor (Correo electrónico: chipres99@ hotmail.com)

$\dagger$ Investigador contribuyendo como primer Autor
} 


\section{Introducción}

Durante la última década, la avicultura se ha convertido en una de las actividades pecuarias de mayor crecimiento. El pollo de engorda es uno de los animales que expresa mayor eficiencia nutricional y productiva, características que se reflejan en la rápida ganancia de peso considerando una absorción eficiente de los nutrientes. Asimismo, el consumo de carne de pollo ha contribuido la seguridad alimentaria de las poblaciones humanas, ofreciendo a los consumidores una fuente de alimento con excelente calidad nutricional (Díaz et al., 2016).

En los últimos años se ha enfatizado el interés por el estudio de los polisacáridos, ya que se ha demostrado que pueden ser considerados como probióticos, por lo que podrían reducir la incidencia de enfermedades. Asimismo, se ha demostrado que estimulan el crecimiento y actividad de bacterias benéficas, y generan respuestas biológicas asociadas a la buena salud del hospedero, por su efecto antiobesogénico, regulador de la glucosa, antioxidante, anticancerígeno e inmunomodulador (MarquezEscalante et al., 2018).

Gutiérrez-Castro \& Corredor-Matus (2017) probaron que el uso de probióticos en la dieta de pollos de engorda genera modificaciones en parámetros del cuadro hemático, con respecto al recuento eritrocitario, concentración de hemoglobina corpuscular media, recuento plaquetario, recuento leucocitario total y el recuento diferencial de monocitos, eosinófilos y linfocitos.

Es de suma importancia la determinación de los parámetros hematológicos en la especie aviar, ya que nos permite evaluar en qué sentido puede estar repercutiendo el producto que se está utilizando y así, profundizar en la investigación correspondiente para evaluar cómo está respondiendo el sistema inmunitario a la afectación en cuestión.

En lo anterior radica la importancia de la determinación de parámetros hematológicos normales en aves para poder evaluar las afectaciones o alteraciones que se generen en la investigación, dependiendo a su vez, de las condiciones de alojamiento y el manejo de las aves (Avilez Colón et al., 2015).
Los valores hematológicos pueden variar por el estado nutricional, sexo, edad, hábitat, época del año, estado reproductivo y estrés; por lo tanto, es importante que exista un equilibrio entre las células hemáticas para mejorar la condición fisiológica de las aves y la respuesta celular. Se ha demostrado que los probióticos ejercen un efecto como adyuvantes inmunes modulando la respuesta inmune en la mucosa, modulando la respuesta inflamatoria, estimulando la producción de lagunas citoquinas y la actividad fagocítica de macrógafos y neutrófilos, y mejorando las respuestas de anticuerpos específicos (Gutiérrez-Castro \& Corredor-Matus, 2017).

Pizarro et al. (2014) estudiaron que los polisacáridos tienen efectos positivos sobre la inmunidad del huésped, previniendo los riesgos de infecciones o cáncer, aunque también mencionan que se desconoce el efecto de su respuesta a largo plazo. A su vez, Cardoso et al. (2018) mencionan que los polisacáridos mejoran el valor nutritivo de la dieta para pollos de engorda.

Por lo anterior, el propósito de este trabajo fue la evaluación del efecto de polisacáridos en parámetros productivos y a su vez, en sistema inmunológico de las aves, con el objetivo de probar si existe un efecto que vuelva más resistentes a las aves contra los patógenos, sometiendo a las mismas, a condiciones de estrés para sugerir un efecto inmunoestimulante.

\section{Materiales y métodos}

El presente trabajo se desarrolló en la caseta de pollos de engorda del Departamento de Producción Animal del Centro Universitario de Ciencias Biológicas y Agropecuarias de la Universidad de Guadalajara ubicado en Camino Ramón Padilla Sánchez No. 2100 Nextipac, Zapopan, Jalisco con las coordenadas $20^{\circ} 74^{\prime} 59.05^{\prime \prime}$, de latitud Norte, $103^{\circ} 50^{\prime} 96.38^{\prime \prime}$ de longitud Oeste y altitud de $1670 \mathrm{~m}$.

Se utilizaron 582 pollos machos de línea genética Cobb, con un día de nacidos los cuales fueron distribuidos en 2 tratamientos con 3 repeticiones de 97 aves: tratamiento control (T) y tratamiento con polisacáridos $(\mathrm{P})$. Los polisacáridos utilizados fueron de la marca Olmix@ y se utilizaron a una dosis de $45 \mathrm{~g}$ por cada $500 \mathrm{~L}$ de agua los días 9, 11, 12, 19, 21, 22, 34,36 , y 37 de vida. 
Se alojaron en corrales de $9.7 \mathrm{~m}^{2}$. A ambos tratamientos se les aplico vacuna contra gumboro en el agua de bebida a los días 2 y 14 de vida, así como la vacuna contra Newcastle vía oftálmica los días 10 y 20. Los muestreos para el recuento de leucocitos y linfocitos fueron realizados los días $9,12,19,22,34$ y 37 . Se determinaron parámetros productivos mediante el pesaje de rechazos de alimento de forma semanal en una báscula marca OHAUS ${ }^{\circledR}$ modelo T21P y se obtuvo el consumo promedio, se pesó a cada animal de forma individual una vez por semana y se determinó ganancia de peso.

La conversión alimenticia se obtuvo mediante la relación del alimento consumido por corral y la ganancia de peso promedio por corral. Para la medición de la respuesta celular, se realizó frotis teñido con la técnica hayem-turk para posteriormente realizar el conteo en la cámara de neubauer. Los datos fueron analizados mediante un ANDEVA y la comparación de medias fue analizada por el método de Fisher. Para analizar los datos se utilizó el programa Minitab 18 Copyright 2017 ®.

\section{Resultados y discusión}

\section{Consumo de alimento}

En la tabla 1 se muestran los consumos semanales que tuvieron las aves durante el proyecto, no existió una diferencia significativa ( $\mathrm{p}>0.05$ ) en las 7 semanas, resultados que concuerdan con los obtenidos por Kang et al., (2013) donde probaron un tratamiento adicionado con antibioticos y tres con diferentes presentaciones del alga Chlorella vulgaris y ninguno obtuvo un consumo diferente al tratamiento control, así mismo Abudabos et al., (2013) probaron el alga Ulva lactuca en dos concentraciones distintas y tampoco obtuvieron diferencias. Se puede observar que la tendencia en el tratamiento con polisacáridos fue un consumo mayor de alimento, únicamente en la semana 2 , el tratamiento $\mathrm{T}$ consumió $4 \mathrm{~g}$ más que el tratamiento $P$, en la sexta semana la diferencia de consumo fue más marcada, siendo esta de 25 $\mathrm{g}$ más en el tratamiento $\mathrm{P}$, datos que coinciden con valores obtenidos por Evans et al., (2015) donde probaron diferentes porcentajes de inclusión del alga Spirulina y los valores no son diferentes a los obtenidos por el tratamiento de alimento comercial.
Sin embargo la máxima inclusión de esta alga logro tener un menor consumo $(\mathrm{p}<0.05)$ que el resto de los tratamientos. De acuerdo con los estudios citados anteriormente se muestra que solo la concentración más alta tuvo un efecto en el consumo de alimento, por lo cual se tiene que valorar el costo beneficio de agregar porcentajes tan altos de aditivos.

\begin{tabular}{|c|c|c|c|c|c|}
\hline \multicolumn{2}{|c|}{ Tratamiento } & $\mathbf{T}$ & $\mathbf{P}$ & E.E. & $\begin{array}{c}\text { Valor } \\
\mathbf{P}\end{array}$ \\
\hline \multirow{7}{*}{ 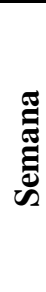 } & 1 & 111 & 112 & 5.2 & 0.915 \\
\hline & 2 & 262 & 258 & 5.2 & 0.664 \\
\hline & 3 & 662 & 673 & 5.2 & 0.287 \\
\hline & 4 & 904 & 920 & 11.1 & 0.465 \\
\hline & 5 & 1027 & 1031 & 31.1 & 0.941 \\
\hline & 6 & 1321 & 1346 & 21.6 & 0.539 \\
\hline & 7 & 1468 & 1470 & 21.4 & 0.978 \\
\hline
\end{tabular}

Tabla 1 Consumo de alimento semanal en g por ave

\section{Ganancia de peso}

En la tabla 2, se observa la ganancia de peso que obtuvieron las aves semanalmente, en la primera semana el tratamiento $\mathrm{T}$ obtuvo mayor ganancia de peso con una diferencia de 2 gramos, en la semana dos y tres, el tratamiento $\mathrm{P}$ gano mayor peso, sin embargo la diferencia no fue mayor a 3 gramos, a partir de la semana cuatro se obtuvo una mayor diferencia en el peso del tratamiento $\mathrm{P}$, siendo la semana siete la que consiguió la diferencia más amplia, con 77 gramos más sin embargo las diferencias no fueron significativas ( $p>0.05$ ), siendo estos resultados semejantes a los obtenidos por Rezvani et al., (2012), donde en su estudio realizado probaron diferentes concentraciones del alga Chlorella vulgaris y prebióticos y en ninguno de sus tratamientos existio diferencia contra el tratamiento control el cual no contaba con aditivos, Evans et al., (2015) ensayaron con diferenes porcentajes de inclusión del alga Spirulina y Abudabos et al., (2013) que adicionaron Ulva lactuca en dos concentraciones, tampoco lograron mostrar una mayor ganancia de peso.

Caso contrario al estudio realizado por Kang et al., (2013) donde los tratamientos adicionados con alga obtuvieron mayor ganancia de peso ( $\mathrm{p}<0.05)$, de igual manera al estudio de Gutiérrez et al., (2015) donde se utilizaron probióticos. 


\begin{tabular}{|c|r|r|r|r|r|}
\hline \multicolumn{2}{|c}{ Tratamiento } & \multicolumn{1}{c}{ T } & \multicolumn{1}{c|}{ P } & \multicolumn{1}{c|}{ E.E. } & Valor P \\
\hline \multirow{4}{*}{} & $\mathbf{1}$ & 168 & 164 & 1.96 & 0.305 \\
\cline { 2 - 6 } & $\mathbf{2}$ & 420 & 423 & 5.86 & 0.759 \\
\cline { 2 - 6 } & $\mathbf{3}$ & 835 & 836 & 4.43 & 0.967 \\
\cline { 2 - 6 } & $\mathbf{4}$ & 1347 & 1378 & 17.53 & 0.361 \\
\cline { 2 - 6 } & $\mathbf{5}$ & 1840 & 1896 & 31.44 & 0.357 \\
\cline { 2 - 6 } & $\mathbf{6}$ & 2600 & 2677 & 64.34 & 0.528 \\
\cline { 2 - 6 } & $\mathbf{7}$ & 3252 & 3301 & 101.59 & 0.792 \\
\hline
\end{tabular}

Tabla 2 Ganancia de peso por ave en $g$

\section{Conversión alimenticia}

En la conversión alimenticia se puede observar que fue mejor la del tratamiento $\mathrm{T}$ con un valor de 2.267, aunque la diferencia no es significativa ( $\mathrm{p}>0.05$ ), resultados coincidentes con los estudios realizados por Kang et al., (2013), Evans et al., (2015) y Gutiérrez et al., (2015) donde no existió diferencia en los tratamientos adicionados con algas, tanto el presente estudio como los antes mencionados difieren con el realizado por Rezvani et al., (2012) donde el tratamiento control fue el que presento la conversión alimenticia más alta comparado con los tratamientos con Chlorella vulgaris y prebióticos que obtuvieron una mejor conversión, estudio que coincide con el realizado por Gutiérrez et al., (2015) donde el tratamiento con probióticos reporto una conversión más baja. Estas diferencias pueden deberse a las concentraciones utilizadas en los distintos estudios, así como también a la presentación de los aditivos y la línea genética de aves utilizada en estos.

\begin{tabular}{|l|l|l|l|}
\hline T & P & E.E. & Valor P \\
\hline 2.267 & 2.388 & 0.121 & 0.596 \\
\hline
\end{tabular}

Tabla 3 Conversión alimenticia promedio por tratamiento

\section{Leucocitos y linfocitos}

Los valores obtenidos de los leucocitos se muestran en la tabla 4, en la muestra de los días 9,12 y 19 se observa que las aves suplementadas con polisacáridos muestran una mayor cantidad de glóbulos blancos, sin existir diferencia significativa ( $p>0.05)$, sin embargo, el día 37 el tratamiento $\mathrm{P}$ fue mayor con una $\mathrm{p}$ de 0.06 . El día 22 los valores de ambos tratamientos fueron $2.16 \times 10^{9} / 1$, la muestra del día 34 fue mayor por 0.19 en el tratamiento $\mathrm{T}$, que fue la única semana en que este fue más alto.
Los linfocitos se muestran en la tabla 5, donde se puede observar que en los días 9, 22 y 37 fueron más altos en el tratamiento adicionado con polisacáridos y en los días 12, 19 y 34 en el tratamiento $\mathrm{T}$, se observa también que en el día 22 la diferencia de 0.26 entre los tratamientos fue significativa ( $\mathrm{p}<0.05)$, el estudio realizado por Eyng et al., (2015) muestra que 5 concentraciones distintas de extracto etanólico de propóleos no genera una diferencia en la cantidad de linfocitos contados contra el tratamiento comercial, en el estudio realizado por Gutiérrez-Castro \& Corredor-Matus (2017) donde probaron distintos probióticos obtuvieron recuentos leucocitarios menores al tratamiento control, sin embrago en el recuento linfocitario no encontraron diferencia significativa ( $\mathrm{p}>$ $0.05)$.

Kang et al., (2013), obtuvieron una diferencia significativa $(\mathrm{p}<0.05)$ entre los tratamientos con extracto líquido de Chlorella el cual obtuvo mayor cantidad de leucocitos y linfocitos que el tratamiento con Chlorella seca, sin embargo, ambos tratamientos fueron iguales ( $p>0.05$ ) que el tratamiento control, el cual no contaba con aditivos. Esto demuestra que la presentación o la forma de extraer los polisacáridos puede afectar en los resultados del experimento.

\begin{tabular}{|r|c|c|c|r|} 
Días & $\mathbf{T}$ & \multicolumn{1}{|c}{ P } & E.E. & Valor de P \\
\hline 9 & 1.56 & 1.62 & 0.05 & 0.55 \\
\hline 12 & 1.69 & 1.89 & 0.08 & 0.163 \\
\hline 19 & 2.81 & 2.88 & 0.12 & 0.764 \\
\hline 22 & 2.16 & 2.16 & 0.03 & 1 \\
\hline 34 & 2.85 & 2.66 & 0.11 & 0.370 \\
\hline 37 & 2.72 & 2.89 & 0.05 & 0.067 \\
\hline
\end{tabular}

Tabla 4 Leucocitos $\times 10^{9} / 1$

\begin{tabular}{|r|r|r|r|r|} 
Días & \multicolumn{1}{l}{ T } & \multicolumn{1}{c|}{ E.E. } & Valor de P \\
\hline 9 & 0.94 & 1.06 & 0.042 & 0.116 \\
\hline 12 & 0.99 & 1.21 & 0.085 & 0.165 \\
\hline 19 & 2.21 & 2.11 & 0.109 & 0.619 \\
\hline 22 & $1.39^{\mathrm{a}}$ & $1.65^{\mathrm{b}}$ & 0.044 & 0.002 \\
\hline 34 & 1.49 & 1.36 & 0.075 & 0.312 \\
\hline 37 & 1.56 & 1.75 & 0.106 & 0.304 \\
\hline
\end{tabular}

Tabla 5 Linfocitos $\times 10^{9} / 1$

\section{Conclusiones}

Los polisacáridos de alga marina no mostraron efectos sobre el comportamiento productivo.

La respuesta leucocitaria y linfocitaria no mostró cambios con la adición de polisacáridos. 
Se debería probar dosificaciones distintas de polisacáridos de alga marina para poder obtener conclusiones mas contundentes.

\section{Referencias}

Abudabos, A. M., Okab, A. B., Aljumaah, R. S., Samara, E. M., Abdoun, K. A., \& Al-Haidary, A. A. (2013). Nutritional value of green seaweed (Ulva lactuca) for broiler chickens. Italian Journal of Animal Science, 12(2), 177-181. https://doi.org/10.4081/ijas.2013.e28

Avilez Colón, B. L., Rugeles Pinto, C. C., Jabib Ruiz, L., \& Herrera Benavides, Y. M. (2015). Parámetros hematológicos en pollos de engorde criados en una granja de producción cerrada en el trópico bajo. Revista de Medicina Veterinaria, 4(29), 33. https://doi.org/10.19052/mv.3444

Cardoso, V., Fernandes, E. A., Santos, H. M. M., Maçãs, B., Lordelo, M. M., Telo da Gama, L., ... Ribeiro, T. (2018). Variation in levels of nonstarch polysaccharides and endogenous endo1,4- $\beta$-xylanases affects the nutritive value of wheat for poultry. British Poultry Science, 59(2), 218-226.

https://doi.org/10.1080/00071668.2018.142367 4

Díaz, E. A., Narváez-Solarte, W., \& Giraldo, J. A. (2016). Alteraciones Hematológicas y Zootécnicas del Pollo de Engorde bajo Estrés Calórico. Informacion Tecnologica, 27(3), 221$230 . \quad$ https://doi.org/10.4067/S071807642016000300021

Evans, A. M., Smith, D. L., \& Moritz, J. S. (2015). Effects of algae incorporation into broiler starter diet formulations on nutrient digestibility and 3 to $21 \mathrm{~d}$ bird performance. Journal of Applied Poultry Research, 24(2), 206-214. https://doi.org/10.3382/japr/pfv027

Eyng, C., Murakami, A. E., Ospina-Rojas, I. C., Pedroso, R. B., Silveira, T. G. V., \& Lourenço, D. A. L. (2015). Efecto de la inclusión dietÃtica de extracto etanólico de propóleos en la inmunidad de pollos de engorde. Archivos de Medicina Veterinaria, 47(2), 185-192.

Gutiérrez-Castro, L. L., \& Corredor-Matus, J. R. (2017). Parámetros sanguíneos y respuesta inmune en pollos de engorde alimentados con probióticos. Veterinaria y Zootecnia, 11, 81-92. https://doi.org/10.17151/vetzo.2017.11.2.7
Gutiérrez, L., Bedoya, O., \& Arenas, J. (2015). Evaluación de parámetros productivos en pollos de engorde suplementados con microorganismos probióticos. Temas Agrarios, 20(2), 81. https://doi.org/10.21897/rta.v20i2.761

Kang, H. K., Salim, H. M., Akter, N., Kim, D. W., Kim, J. H., Bang, H. T., ... Suh, O. S. (2013). Effect of various forms of dietary Chlorella supplementation on growth performance, immune characteristics, and intestinal microflora population of broiler chickens. Journal of Applied Poultry Research, 22(1), $100-108$. https://doi.org/10.3382/japr.2012-00622

Marquez-Escalante, J., Carvajal-Millan, E., López-Franco, Y. L., Valenzuela-Soto, E. M., Rascón-Chu, A., \& Escalante, J. M. (2018). Prebiotic effect of Arabinoxylans and Arabinoxylan-Oligosaccharides and the relationship with good health promotion Efecto prebiótico de los Arabinoxilanos y los Arabinoxilo-Oligosacáridos y su relación con la promoción de la buena salud. CienciaUAT, 146(1), $146-164$ https://doi.org/10.29059/cienciauat.v13i1.922

Pizarro, S., Ronco, A. M., \& Gotteland, M. (2014). Betaglucanos Que Tipos Existen Y Sus Beneficios. Revista Chilena de Nutrición, 41(3), 439-446.

Rezvani, M., Zaghari, M., \& Moravej, H. (2012). A survey on Chlorella vulgaris effect's on performance and cellular immunity in broilers. International Journal of Agricultural Science and Research, 3(0), 9-15. 